\title{
Propuesta metodológica para la evaluación de estructuras de madera en monumentos históricos - Caso Hotel El Comercio
}

\author{
Methodological proposal for evaluation of structural wood timber in historic \\ buildings - Case Hotel El Comercio
}

\author{
Daniella Vizcarra Salazar ${ }^{1, \star}$, José Cano Delgado ${ }^{1}$, Luis Yoza Yoza ${ }^{1}$ \\ y Moisés Acevedo Mallque ${ }^{1}$
}

\begin{abstract}
Resumen
El objetivo del estudio fue proponer una metodología para la evaluación de elementos estructurales de madera antigua y estimar la clase resistente actual de los mismos. Se utilizaron dos técnicas para la evaluación de los trece elementos de madera, la inspección visual y métodos no destructivos con equipo de ultrasonido, extractor de tornillo y resistógrafo. Se evaluaron cuatro normas de clasificación visual: NTP 251.104, UNE 56.544, NF B 52-001 y DIN 4074; y se determinó el módulo de elasticidad y módulo de rotura en probetas mediante métodos destructivos y no destructivos en laboratorio. Los resultados obtenidos en obra en base a la inspección visual de los elementos y la inspección de cabezales destapados, en el caso de las vigas, fueron utilizados para formular un criterio de clasificación de acuerdo al grado de ataque biótico: C1 (deterioro $\leq 25 \%)$, C2 (25\% < deterioro $<50 \%$ ) y C3 (deterioro $\geq 50 \%$ ). Asimismo, con el mismo criterio se formuló el porcentaje residual del módulo de elasticidad y el módulo de rotura para clasificar los elementos en: $\mathrm{C} 1=$ Bueno, $\mathrm{C} 2=$ Regular y C3=Malo. Se concluyó que las vigas, viguetas y columnas evaluadas disminuyeron su resistencia en un 50\% del Grupo Estructural C. La metodología desarrollada puede ser tomada como referencia para la evaluación de estructuras de madera antigua in situ.
\end{abstract}

Palabras clave: monumento histórico, evaluación de estructuras de madera, inspección visual, métodos no destructivos, ultrasonido.

\footnotetext{
${ }^{1}$ Departamento de Industrias Forestales, Facultad de Ciencias Forestales, Universidad Nacional Agraria La Molina (UNALM), Av. La Molina s/n, La Molina, Lima, Perú.

* Autor de Correspondencia: daniella.vizsa@gmail.com
} 


\begin{abstract}
The objective of the study was to propose a methodology for the evaluation of structural elements of old wood and to estimate the current resistant class of them. Two techniques were used for the evaluation of the thirteen wooden elements: visual inspection and non-destructive methods with ultrasound equipment, screw extractor and resistograph. Four standards of visual classification were evaluated: NTP 251.104, UNE 56.544, NF B 52-001 and DIN 4074; and the modulus of elasticity and modulus of rupture in specimens was determined by destructive and non-destructive methods in the laboratory. The results of the visual inspection in situ and the inspection of the beam end supports uncovered, was used to formulate a classification criterion according to the degree of biotic attack: C1 (deterioration $\leq 25 \%), \mathrm{C} 2(25 \%<$ deterioration $<50 \%)$ and C3 (deterioration $\geq 50 \%$ ). Likewise, with the same criterion, the residual percentage of the modulus of elasticity and the modulus of rupture were formulated to classify the elements into: $\mathrm{C} 1=\mathrm{Good}, \mathrm{C} 2=$ Regular and C3 = Bad. It was concluded that the beams, joists and columns evaluated reduced their resistance by $50 \%$ of the Structural Group C. The methodology developed can be taken as a reference for the evaluation of old wooden structures in situ.
\end{abstract}

Key words: historical monument, evaluation of wooden structures, visual inspection, non-destructive methods, ultrasound.

\section{Introducción}

La conservación del patrimonio cultural está considerada como un principio fundamental en la vida cultural de las sociedades modernas (Lombillo y Villegas 2006), pero la falta de aplicación de políticas de conservación, agudiza con el pasar del tiempo el deterioro de las mismas. En el Perú, uno de las principales áreas prioritarias para la conservación se encuentra en el centro histórico de Lima reconocida por la UNESCO desde 1991 como patrimonio cultural de la humanidad. Uno de los monumentos históricos que la constituyen es el hotel El Comercio, arquitectura republicana del siglo XIX conformado por materiales de adobe, quincha y un sistema de techos de madera, declarado intangible por su belleza y valor histórico (INC 2007).

En este contexto, la utilización de métodos no destructivos como la inspección visual y los equipos de ultrasonido, extractor de tornillo y resistógrafo, para la evaluación de estructuras de madera antigua in situ, son fundamentales para que el grado de intervención sea mínimo. Respecto a la inspección visual, Esteban (2003) menciona que las normas de clasifi- cación visual de la madera para uso estructural están basadas en investigaciones llevadas a cabo sobre piezas estructurales de madera de pequeña escuadría y de sección regular, en buen estado de conservación, de especie y procedencia conocidas y sometidas a ensayos en condiciones controladas de laboratorio. Estas condiciones de estudio se alejan bastante de las que aparecen en estructuras antiguas, empezando porque las piezas se encuentran colocadas en obra y no es posible desmontarlas para su estudio. La mayoría de las piezas son de escuadría superior e irregular y los daños diversos y ataques de insectos y hongos xilófagos aparecen con frecuencia. En definitiva, existe una gran incertidumbre en los trabajos de peritación de las estructuras.

En relación a la aplicación de técnicas no destructivas, el análisis de los datos obtenidos in situ el estudio de Sotomayor y Cruz (2004) sugieren el cálculo del MOE residual y el MOR residual como indicadores para la determinación de la disminución de densidad y resistencia mecánica de la madera en obra. Asimismo, Arriaga et al. (2002) indican que el fundamento físico que existe entre la velocidad de propagación de onda con la densidad 
permite determinar el módulo de elasticidad. Esta afirmación se refuerza con lo mencionado por Benítez (2009), que existen muchos estilos sobre la aplicación de la técnica de ultrasonido para determinar el estado y la calidad de la madera, y en la gran mayoría el coeficiente de determinación que existe entre los valores de MOE dinámico y MOE estático es mayor al 70 por ciento. Además, el mismo autor cita los siguientes coeficientes de correlación: $\mathrm{R}^{2}=0,72$ (Acuña et al. 2010); $\mathrm{R}^{2}=0,74$ (Íñiguez 2007).

Referente al extractor de tornillos, Basterra et al. (2006), encontraron una relación estadísticamente significativa (con un grado de confianza del 95 por ciento), entre la densidad media de las viguetas y la fuerza media de arranque obtenida en cada uno de ellas. Otro equipo no destructivo que complementó la inspección visual de las estructuras de madera es el resistógrafo, el cual brinda gráficas del perfil de la estructura donde se observan picos altos y bajos los cuales permiten el cálculo del porcentaje de pérdida de resistencia de la madera a la penetración del taladro.

En la presente investigación, se estudiaron las vigas, viguetas y columnas del primer piso del Hotel El Comercio. El objetivo del estudio fue proponer una metodología para la evaluación de elementos estructurales de madera antigua y estimar la clase resistente actual de los mismos.

\section{Materiales y Métodos}

La investigación se realizó sobre un total de 13 elementos estructurales de madera procedentes de la primera planta del Hotel El Comercio (ver Figura 1), ubicado en la intersección del Jirón Ancash y Jirón Carabaya en el distrito de Cercado de Lima (Perú). Los elementos estructurales mencionados consistieron en cuatro vigas de sección 25 x $25 \mathrm{~cm}$ y longitud de 4 a $5 \mathrm{~m}$, cinco viguetas de sección 9 $\mathrm{x} 15 \mathrm{~cm}$ y longitud de $2 \mathrm{~m}$ en promedio y cuatro columnas de $23 \mathrm{~cm}$ de diámetro y $4 \mathrm{~m}$ de longitud en promedio.

Los elementos de madera seleccionados fueron evaluados in situ durante el 2014 - 2016, y se eligieron de acuerdo al criterio de accesibilidad a la estructura y limpieza de la zona.

\section{Metodología para la elaboración de propues- ta metodológica}

Para la elaboración de la propuesta metodológica para la evaluación de estructuras de madera antiguas se tomó como referencia el trabajo realizado por Arriaga et al. (2002), donde plantean las pautas de evaluación como la inspección visual, la identificación de agentes xilófagos, la utilización de equipos no destructivos, la estimación de la resistencia de la madera y las particularidades de estos elementos de madera antigua. Para la clasificación de visual de las piezas utiliza la normativa europea (UNE 56.544, DIN 4074 y NF B 52-001) $y$ tomando en cuenta los agentes xilófagos que se desarrollan en esos países, por lo que para su aplicación en nuestro país se procedió a homologar la norma nacional con las extranjeras para obtener una clasificación visual que sea propicia para la evaluación de maderas antiguas en nuestros monumentos históricos.

Luego se procedió a aplicar las pautas de evaluación definidas por Arriaga et al. (2002) para la obtención de información cualitativa y cuantitativa, esta última obtenida de la aplicación de los equipos no destructivos y que complementarían la parte cualitativa de la evaluación. Para ello se realizó el cálculo de los valores MOE y MOR que serían utilizados para la estimación de la resistencia de las estructuras de madera. Las gráficas obtenidas de la aplicación del resistógrafo se utilizaron como respaldo de la inspección visual.

Finalmente, se procedió a interpretar los resultados cualitativos (inspección visual y gráficas del resistógrafo) y cuantitativos (MOE y MOR) para la obtención del diagnóstico del estado actual de cada elemento estructural (ver Cuadro 1, 2 y 3 ).

\section{Identificación de la madera}

La identificación anatómica de los elementos de madera, realizados por el Laboratorio de Anatomía de la Madera del Departamento Académico de Industrias Forestales de la 

en monumentos hitóricos - Caso Hotel El Comercio

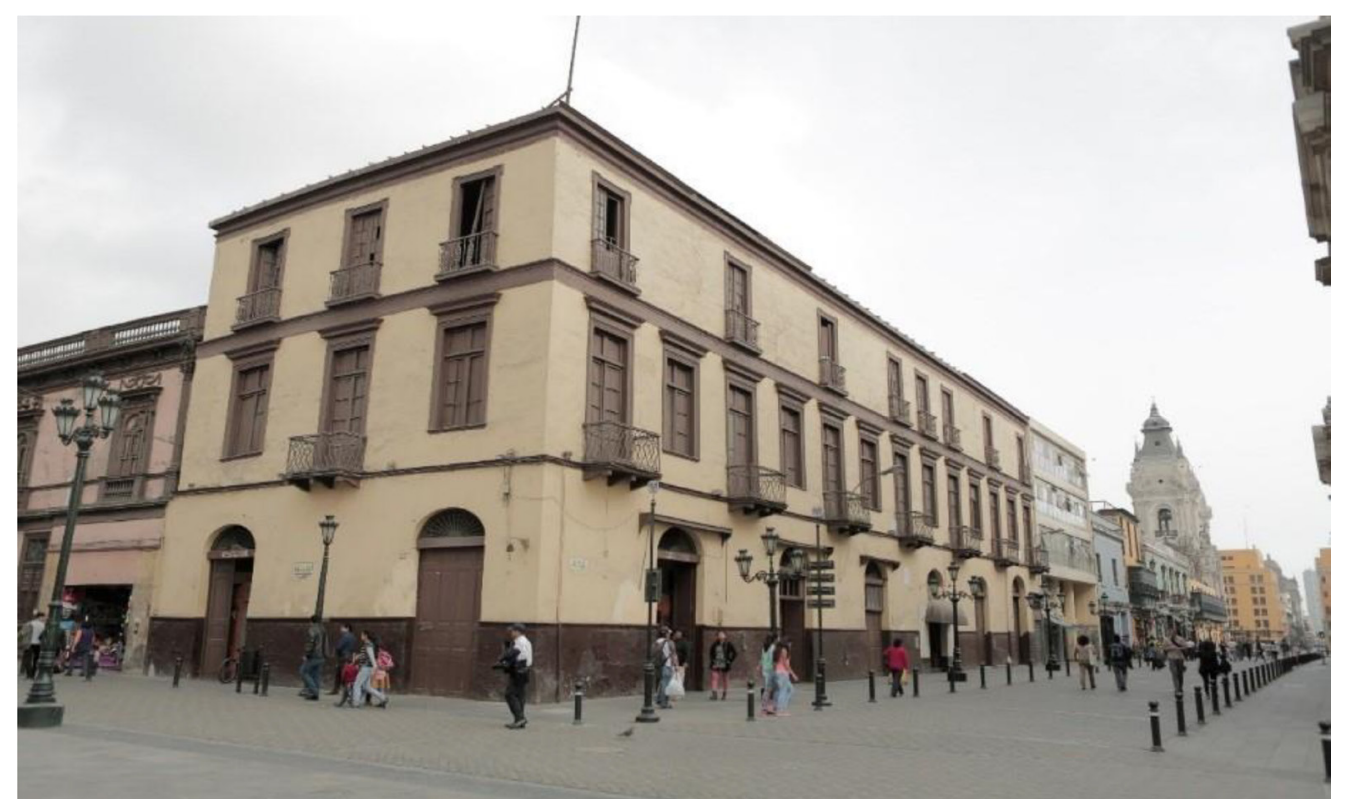

Figura 1. Frente del Hotel El Comercio, Centro de Lima.

Facultad de Ciencias Forestales de la Universidad Nacional Agraria La Molina (UNALM), corroboraron que las muestras corresponden a la especie Entandrophragma sp. (Meliaceae), llamada comúnmente "Caoba africana" o "Sapelli" (Chavesta et al. 2012).

\section{Inspección Visual de la madera}

Previo a la inspección se midió el contenido de humedad con un detector eléctrico de resistencia, procediéndose luego a la inspección visual a lo largo de los elementos de madera. En cada elemento se ubicaron e identificaron las grietas y rajaduras y los ataques bióticos. Para ello se utilizaron punzón, espátula, martillo y formón, para evaluar si existía degradación debajo de la superficie externa de la madera.

Debido a que no existe una normativa para la inspección visual de maderas antiguas se procedió a homologar cuatro normas de clasificación visual de la madera (UNE 56.544, DIN 4074, NF B 52-001 y NTP 251.104), con el fin de obtener una referencia de los parámetros restrictivos a tomar en cuenta en la ins- pección visual de las piezas. Se puede observar el cuadro comparativo de dichas normas (Cuadro 4).

Para la inspección visual de los cabezales de las vigas y viguetas empotrados en los muros de quincha, se realizó el destape de los cabezales accesibles y se registró la magnitud de los defectos solo en la cara frontal debido a que era riesgoso intervenir la pieza quitando material de soporte (ver Figura 2). La inspección visual hizó referencia al estado biótico de la madera, realizándose como primera instancia una clasificación visual de la madera para luego contrastar esta información con los resultados de la aplicación del resistógrafo y llegar a un diagnóstico.

\section{Ensayos no destructivos}

La toma de datos con los equipos no destructivos en vigas se realizó en intervalos de un metro aproximadamente y en el caso de las viguetas en longitud total. El equipo de ultrasonido Fakopp Microsecond Timer de $45 \mathrm{kHz}$ de frecuencia se utilizó para obtener datos sobre la velocidad de propagación en la madera 


\begin{tabular}{|c|c|c|c|c|c|}
\hline \multicolumn{2}{|c|}{ ESTIMACIÓN ACTUAL } & 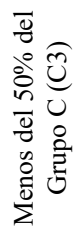 & 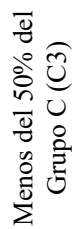 & 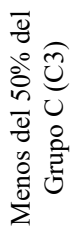 & 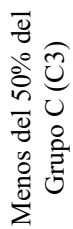 \\
\hline \multirow{3}{*}{ 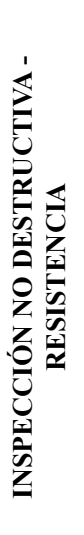 } & DIAGNÓSTICO & 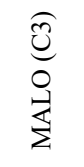 & 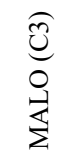 & 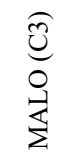 & 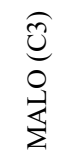 \\
\hline & MOE RESIDUAL & $\underset{\text { త్ర }}{\stackrel{0}{0}}$ & 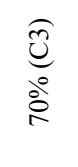 & 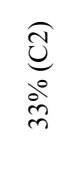 & 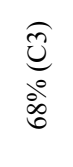 \\
\hline & MOR RESIDUAL & 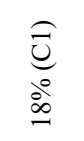 & 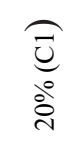 & 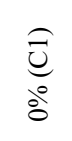 & $\begin{array}{l}\underset{\bar{U}}{0} \\
\stackrel{0}{0}\end{array}$ \\
\hline \multirow{3}{*}{ 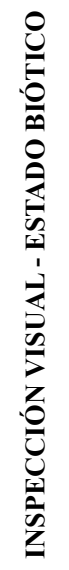 } & DIAGNÓSTICO & 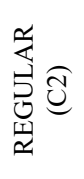 & 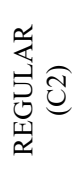 & 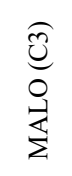 & 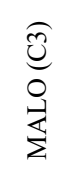 \\
\hline & $\begin{array}{c}\text { GRÁFICAS- } \\
\text { RESISTOGRAFO }\end{array}$ & 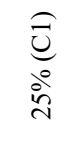 & 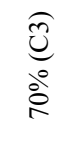 & $\begin{array}{l}\text { 己ֶ } \\
\text { îं } \\
i n\end{array}$ & $\begin{array}{l}\text { త్ } \\
\text { îं } \\
\text { in }\end{array}$ \\
\hline & $\begin{array}{c}\text { CLASIFICACION } \\
\text { VISUAL }\end{array}$ & $\tilde{3}$ & $\bar{U}$ & $\tilde{U}$ & ల̃ \\
\hline \multicolumn{2}{|c|}{$\begin{array}{l}\text { DIMENSIONES } \\
(\mathrm{cm})\end{array}$} & 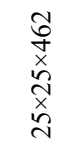 & 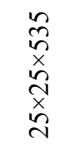 & 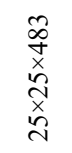 & 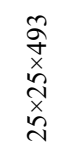 \\
\hline & VIGAS & త্ & 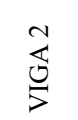 & $\sum_{j}^{m}$ & 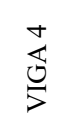 \\
\hline
\end{tabular}



en monumentos hitóricos - Caso Hotel El Comercio

\begin{tabular}{|c|c|c|c|c|c|}
\hline \multicolumn{2}{|c|}{ ESTIMACIÓN ACTUAL } & 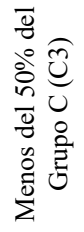 & 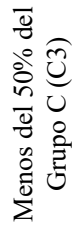 & 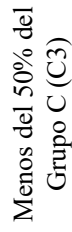 & 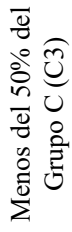 \\
\hline \multirow{3}{*}{ 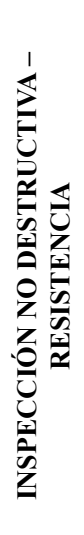 } & DIAGNÓSTICO & 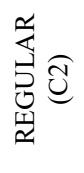 & 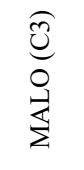 & 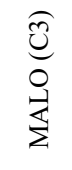 & 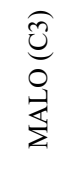 \\
\hline & MOE RESIDUAL & 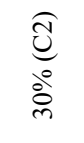 & 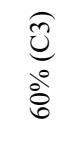 & 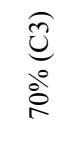 & $\begin{array}{l}\underset{0}{0} \\
\stackrel{\widehat{\sigma}}{\circ}\end{array}$ \\
\hline & MOR RESIDUAL & $\underset{\stackrel{\vec{U}}{0}}{\stackrel{0}{0}}$ & $\begin{array}{l}\text { త্ } \\
\text { in }\end{array}$ & 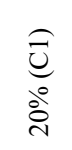 & 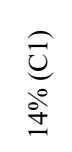 \\
\hline \multirow{3}{*}{ 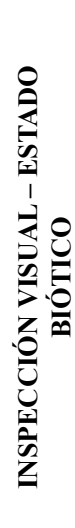 } & DIAGNÓSTICO & 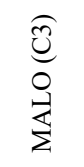 & 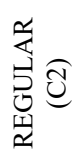 & 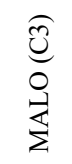 & 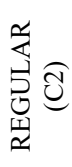 \\
\hline & $\begin{array}{c}\text { GRÁFICAS- } \\
\text { RESISTOGRAFO }\end{array}$ & $\underset{\text { हैं }}{\stackrel{0}{\circ}}$ & $\begin{array}{l}\text { త্ } \\
\text { iें }\end{array}$ & 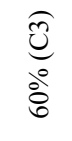 & $\begin{array}{l}\underset{0}{0} \\
\stackrel{0}{\circ}\end{array}$ \\
\hline & $\begin{array}{c}\text { CLASIFICACIÓN } \\
\text { VISUAL }\end{array}$ & U & $\tilde{U}$ & U & $\vec{U}$ \\
\hline \multicolumn{2}{|c|}{$\begin{array}{l}\text { DIMENSIONES } \\
(\mathrm{cm})\end{array}$} & $\begin{array}{l}\stackrel{8}{8} \\
x \\
\dot{x} \\
\text { a }\end{array}$ & $\begin{array}{l}\tilde{J} \\
\infty \\
x \\
\dot{ \pm} \\
x \\
a\end{array}$ & $\begin{array}{l}n \\
0 \\
0 \\
x \\
n \\
x \\
a \\
a\end{array}$ & $\begin{array}{l}\bar{a} \\
\times \\
\stackrel{x}{a} \\
\bar{x}\end{array}$ \\
\hline \multicolumn{2}{|r|}{ VIGUETAS } & 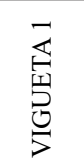 & 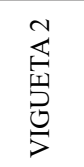 & 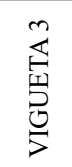 & 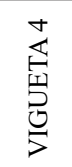 \\
\hline
\end{tabular}




\begin{tabular}{|c|c|c|c|c|c|}
\hline \multicolumn{2}{|c|}{ ESTIMACIÓN ACTUAL } & 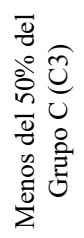 & 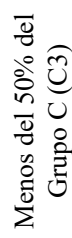 & 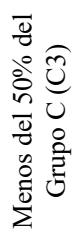 & 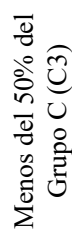 \\
\hline \multirow{3}{*}{ 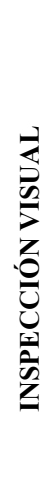 } & DIAGNÓSTICO & 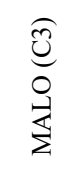 & 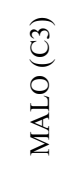 & 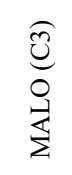 & 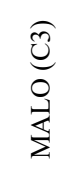 \\
\hline & $\begin{array}{c}\text { GRÁFICAS- } \\
\text { RESISTOGRAFO }\end{array}$ & $\begin{array}{l}\text { త্ } \\
\stackrel{0}{i} \\
i\end{array}$ & 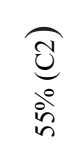 & $\underset{\stackrel{0}{0}}{\stackrel{0}{0}}$ & 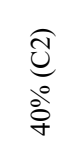 \\
\hline & $\begin{array}{c}\text { CLASIFICACIÓN } \\
\text { VISUAL }\end{array}$ & $\tilde{U}$ & $\tilde{U}$ & $\tilde{U}$ & $\tilde{U}$ \\
\hline \multicolumn{2}{|r|}{$\begin{array}{l}\text { DIMENSIONES } \\
(\mathrm{cm})\end{array}$} & 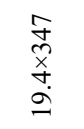 & 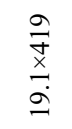 & $\begin{array}{l}\dot{+} \\
\tilde{x} \\
\stackrel{x}{x} \\
\dot{\infty}\end{array}$ & $\begin{array}{l}\tilde{n} \\
\tilde{n} \\
x \\
\infty \\
\infty \\
-\end{array}$ \\
\hline \multicolumn{2}{|r|}{ UBICACIÓN } & 氞 & 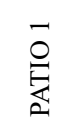 & 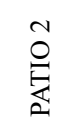 & 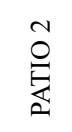 \\
\hline \multicolumn{2}{|r|}{ COLUMNAS } & 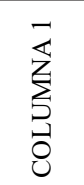 & 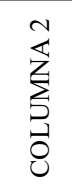 & $\sum_{\substack{\mid \\
ٍ}}^{\infty}$ & 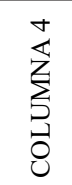 \\
\hline
\end{tabular}

y con ello determinar el módulo de elasticidad (MOE) como indican diversos autores, entre ellos Arriaga et al. (2002), mediante la siguiente ecuación:

$$
\mathrm{E}=v^{2} \times p
$$

Donde:

$\mathrm{E}=$ módulo de elasticidad

$v=$ velocidad de propagación de onda en $\mathrm{m} / \mathrm{s}$ $p=$ densidad en $\mathrm{g} / \mathrm{cm}^{3}$
Con el extractor de tornillo Fakopp Screw Withdrawal Force se midió la fuerza de extracción para estimar la densidad mediante la siguiente ecuación propuesta por Basterra et al. (2006):

$$
\mathrm{D}=1 /(0,93+2,47 / \mathrm{F})
$$

Donde:

$\mathrm{D}=$ densidad en $\mathrm{g} / \mathrm{cm}^{3}$

$\mathrm{F}=$ fuerza al arranque de tornillo en $\mathrm{kN}$ 

en monumentos hitóricos - Caso Hotel El Comercio

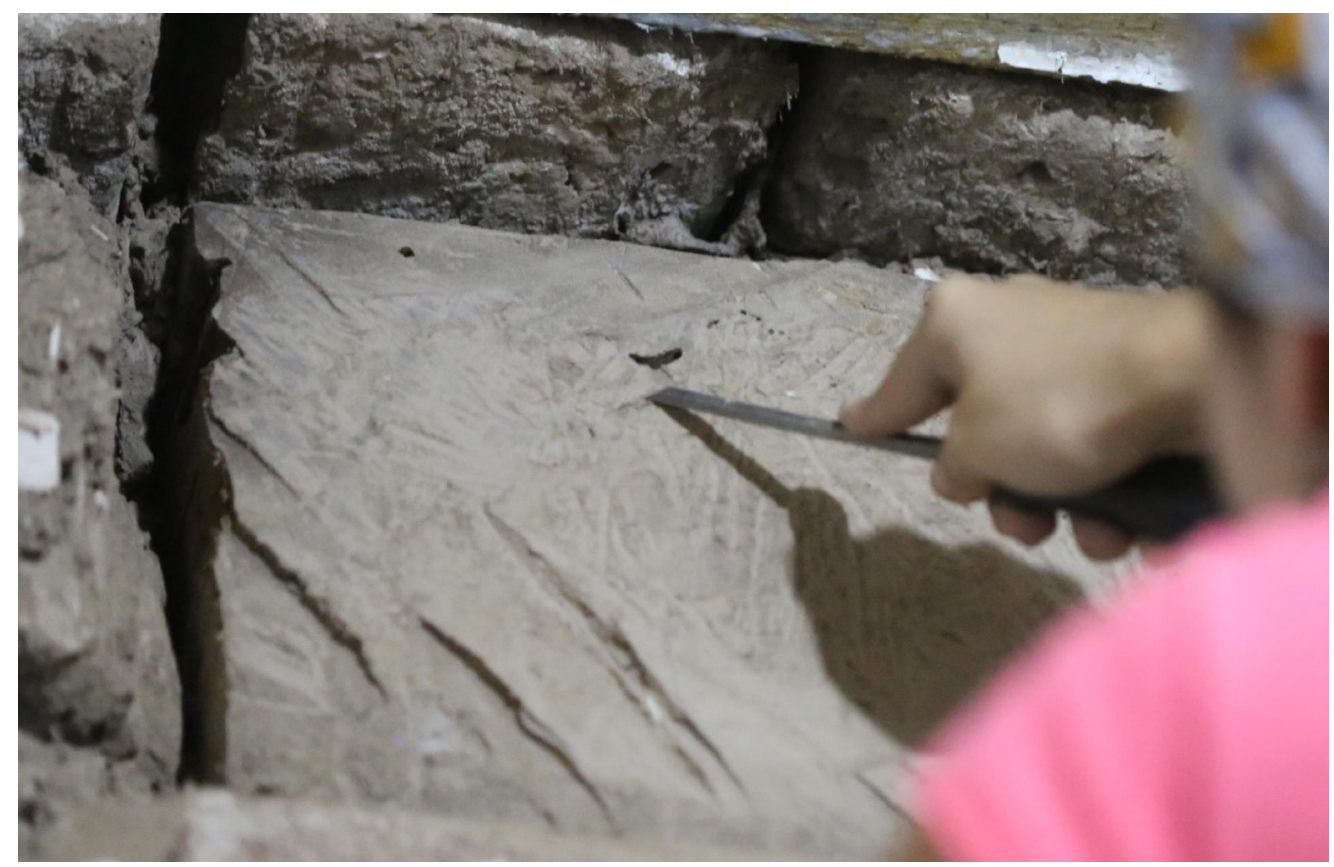

Figura 2. Inspección superficial de cabezales de vigas.

Asimismo, para determinar el módulo de rotura (MOR) se empleó la ecuación citada por Divos et al. (1999), quienes encontraron una relación entre la fuerza de arranque y la velocidad de propagación de onda, la relación encontrada fue la siguiente:

$$
\mathrm{MOR}=1,258 \times \mathrm{F} \times \mathrm{V}^{2}+36,9
$$

Donde:

$\mathrm{MOR}=$ módulo de ruptura en $\mathrm{MPa}$ $\mathrm{F}$ = fuerza al arranque de tornillo en $\mathrm{kN}$ $\mathrm{V}=$ velocidad de propagación de la onda en $\mathrm{m} / \mathrm{s}$

El resistógrafo IML F300 fue utilizado para la inspección del estado biótico generando gráficas del perfil central de cada intervalo de inspección. El resistógrafo evalúa la resistencia que la madera opone a la penetración de una aguja de acero de 1,5 mm de diámetro y de longitud máxima de hasta $30 \mathrm{~cm}$ que gira a velocidad constante mientras se introduce en la madera. El valor de la resistencia del taladro es medido a través de la potencia del motor del taladro, que posteriormente se interpretará en términos de la variación de densidad. El estudio grafico de los perfiles resistográficos aporta una gran información sobre la morfología de la madera, la presencia de nudos, la existencia de galerías producidas por insectos xilófagos en el interior de la madera o de pudriciones (Álvarez et al. 2005).

La información obtenida en vigas y viguetas utilizando el equipo Microtimer second se realizó aplicando tres mediciones, medición cruzada, longitudinal y perpendicular según los intervalos de inspección (Figura 3). Dentro de esos mismos intervalos también se aplicó el extractor de tornillo en las 3 caras de las vigas y viguetas para la obtención de datos de fuerza y la penetración del resistógrafo para obtener gráficas del perfil de la madera. Se aplicó el resistógrafo cerca de los puntos de inserción del extractor de tornillo con dirección de penetración de la cara inferior a la superior con el propósito de conocer el estado de la madera en la cara de la estructura donde no fue posible 


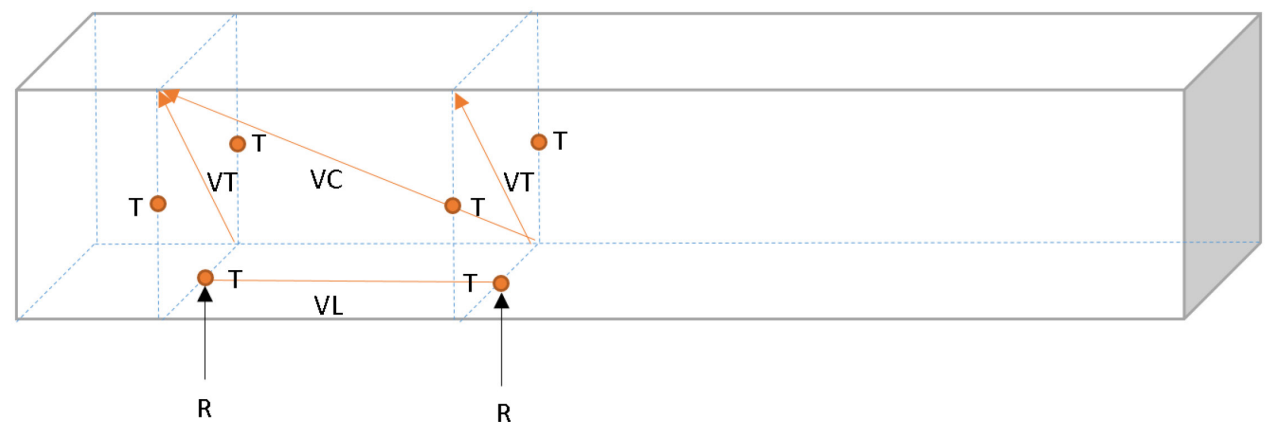

Figura 3. Medición con equipos no destructivos por intervalos. La figura muestra los ensayos aplicados con los tres equipos no destructivos en uno de los cinco intervalos que se determinaron a lo largo de las vigas. Los puntos $\mathrm{T}$ son los puntos de ingreso del extractor de tornillo, los puntos $\mathrm{R}$ son los puntos de penetración del resistógrafo y la dirección de onda del ultrasonido determinaron las velocidades VT (Velocidad Transversal), VC (Velocidad Cruzada y VL (Velocidad Longitudinal).

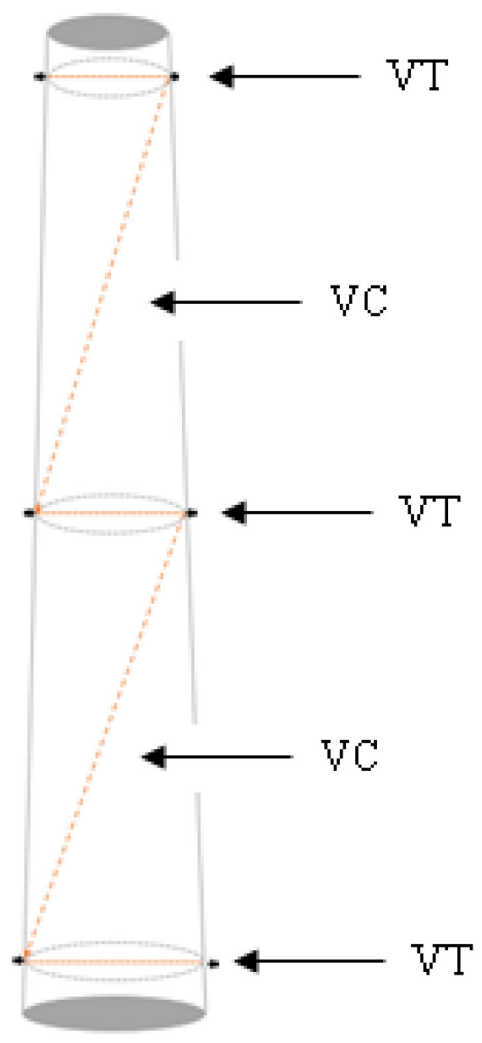

su destape. Se codificó la medición de las velocidades, fuerza y gráficas $(\mathrm{VT}=$ velocidad transversal, $\mathrm{VC}=$ velocidad cruzada, $\mathrm{VL}=$ velocidad longitudinal, $\mathrm{T}=$ extractor de tornillo y $\mathrm{R}=$ resistógrafo).

En relación a las columnas, la toma de datos se realizó aplicando la metodología propuesta por Sandoz y Benoit (2006) agregando la medición cruzada. Se realizaron tres mediciones transversales, a $15 \mathrm{~cm}$ del tope, a la mitad de la columna y a $15 \mathrm{~cm}$ de la base. La medición cruzada se realizó en dos zonas, la mitad superior e inferior de la columna (Figura 4). La dirección de penetración del resistógrafo fue de Este a Oeste, en la misma dirección de la medición de la velocidad transversal, y en tres puntos a lo largo de la columna: parte superior, centro y base. Se codificó la medición de esta velocidad para esta estructura (VT=Velocidad transversal, VC=Velocidad cruzada).

Para la validación de las mediciones con equipos de ultrasonido y extractor de tornillo se realizaron ensayos en laboratorio con probe-

Figura 4. Medición con equipos no destructivos en columnas. Los equipos no destructivos utilizados para la evaluación de columnas fueron el ultrasonido registrando velocidades VT (Velocidad Transversal) y VC (Velocidad Cruzada), y resistógrafo introducido en el mismo sentido que las velocidades VT. 
tas de $2,5 \times 2,5 \times 41 \mathrm{~cm}$ seleccionándose al azar 24 unidades. Las probetas de madera se obtuvieron a partir de un bloque de viga de la especie en estudio que se encontraba desmontada en el lugar de estudio, producto del derrumbe de una zona del primer piso. Se determinó la velocidad de onda y humedad, procediéndose luego a realizar los ensayos destructivos en flexión estática según norma NTP 251.017.2004. Para la validación del MOE dinámico (no destructivo) y MOE estático (destructivo) se determinó $\mathrm{R}^{2}$, utilizando la fórmula propuesta por diversos autores, entre ellos Arriaga et al. (2002).

Los valores estimados por las ecuaciones propuestas por Basterra et al. (2006) para la densidad y el MOR mediante la resistencia al arranque de tornillos (fuerza), son contrastados con los valores obtenidos mediante ensayos destructivos y para su validación se determinó el $\mathrm{R}^{2}$.

En relación a la aplicación de técnicas no destructivas, se tomó en cuenta para el análisis de los datos obtenidos en los elementos de madera in situ el estudio de Sotomayor y Cruz (2004), quienes sugieren el cálculo del MOE residual y el MOR residual como indicadores para la determinación de la disminución de densidad y resistencia mecánica de la madera en obra. Por lo que, se calcularon los parámetros de resistencia MOE y MOR, para luego hacer el análisis residual de dichos valores tomando como referencia los datos obtenidos de madera libre de defectos.

\section{Resultados}

La validación de los equipos de ultrasonido y extractor de tornillo se realizó utilizando el parámetro de módulo de elasticidad y densidad respectivamente. El MOE estimado o estático fue obtenido mediante ensayos en laboratorio y el MOE calculado o dinámico se obtuvo a partir de los datos de los equipos no destructivos. De igual forma, con la densidad estimada y la densidad calculada. El coeficiente de determinación para los valores obtenidos con ultrasonido es de 0.8 (Figura 5) y para los valores obtenidos con extractor de tornillos es de 0.7 (Figura 6).

Respecto a la metodología propuesta para determinar el estado actual de las estructuras de madera antigua in situ, en primer lugar se procedió a homologar la norma nacional con las extranjeras (Cuadro 4), con el fin de encontrar parámetros restrictivos para la clasificación visual de las piezas de madera. Dando como resultado que los parámetros utilizados por dichas normas no eran aplicables para la clasificación visual debido a que se enfocaban en los defectos mecánicos de la madera cómo fendas y grietas, que en la evaluación de las piezas in situ no eran parámetros relevantes, debido a que se observaron pocos de estos defectos en las piezas evaluadas. Se observó que los defectos que tenían más relevancia en las piezas de madera evaluadas eran de origen biótico, lo cual en todas las normas analizadas no tenían relevancia en la clasificación visual. Es por ello que se propone una clasificación de tres categorías: C1 (Bueno), C2 (Regular) y C3 (Malo); teniendo como parámetros restrictivos la inspección visual y la inspección no destructiva que se resume en el Cuadro 5.

En segundo lugar, se procedió a resumir mediante un mapa conceptual los pasos a seguir para una evaluación de estructuras de madera antigua de una forma práctica y de fácil comprensión (Figura 7).

Para la estimación del estado de las maderas evaluadas se tuvo que formular un cuadro de clasificación que indicara los rangos en que los elementos de madera se consideraran en buen estado, estado regular o mal estado debido a que la homologación de las normas de clasificación visual no proporcionó parámetros que se ajustarán a las condiciones vistas en obra. Estas normas no definen rangos de calidad tomando en cuenta el deterioro de la madera por agentes bióticos ni la resistencia residual que fueran útiles para la clasificación de las piezas evaluadas. También se consideró las gráficas del resistógrafo como información relevante debido a que brinda resultados de forma directa e inmediata que son de gran utilidad cuan- 


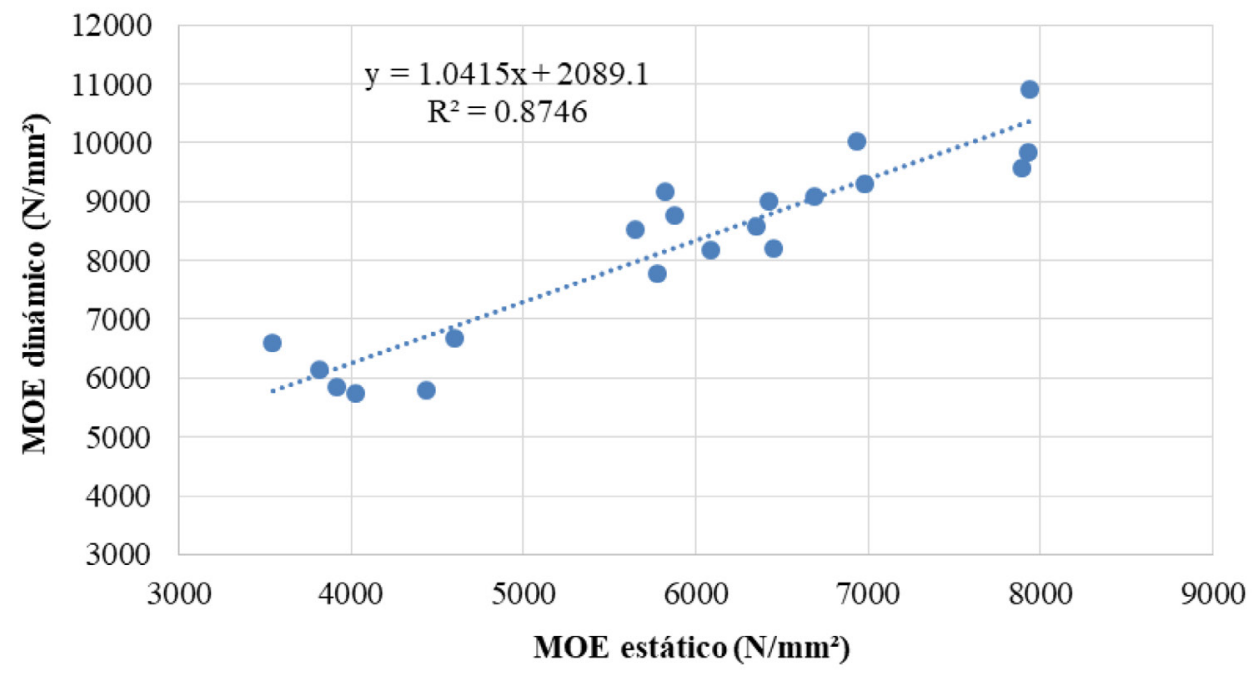

Figura 5. Coeficiente de determinación del MOE dinámico vs. MOE estático.

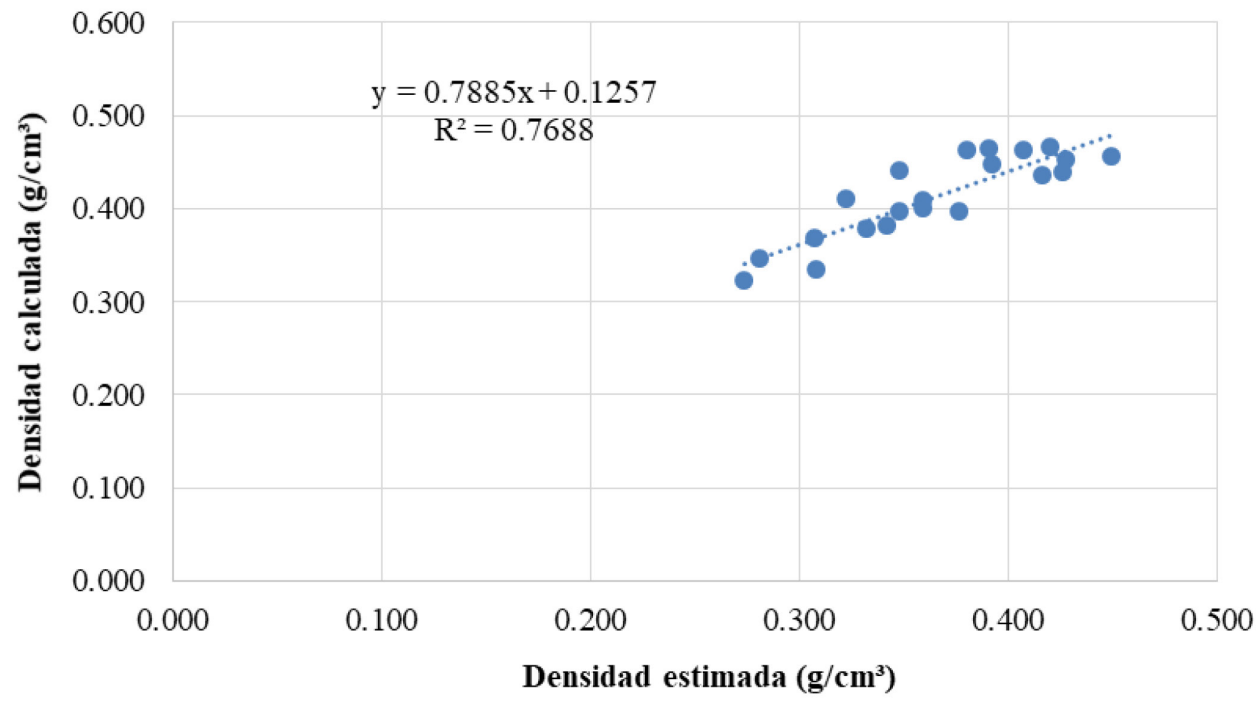

Figura 6. Coeficiente de determinación de la densidad estimada y densidad calculada. 


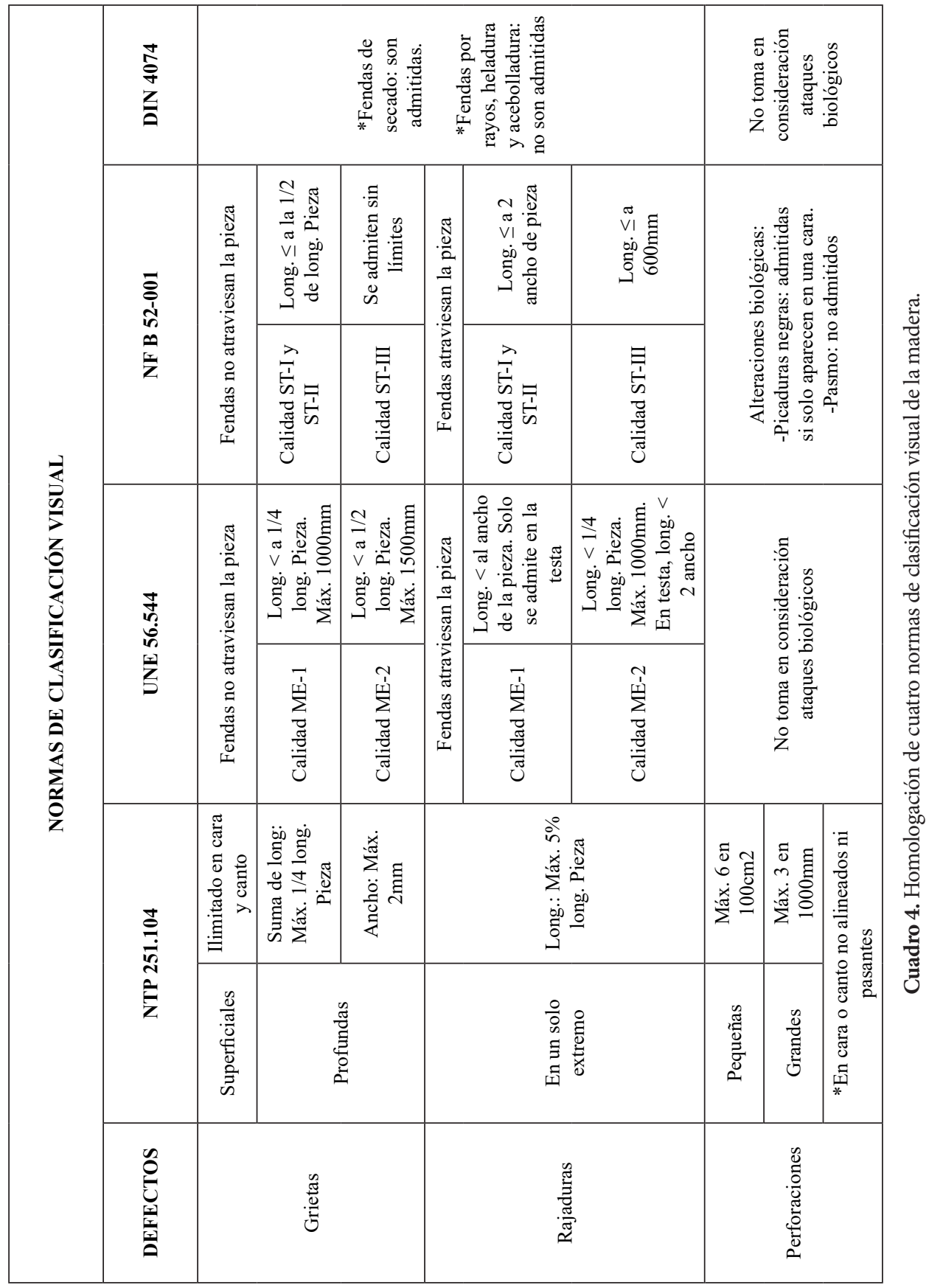




\begin{tabular}{|c|c|c|c|c|}
\hline CLASIFICACIÓN & $\begin{array}{c}\text { INSPECCIÓN } \\
\text { VISUAL }\end{array}$ & RESISTÓGRAFO & $\begin{array}{c}\text { RESISTENCIA } \\
\text { RESIDUAL }\end{array}$ & DIAGNÓSTICO \\
\hline $\mathrm{C} 1$ & $\begin{array}{c}\text { deterioro } \leq 25 \% \\
\text { del área }\end{array}$ & $\begin{array}{c}\text { pérdida de } \\
\text { resistencia } \leq 25 \%\end{array}$ & $\begin{array}{c}\text { resistencia residual } \\
\leq 25 \%\end{array}$ & BUENO \\
\hline $\mathrm{C} 2$ & $\begin{array}{c}25 \% \text { del área }< \\
\text { deterioro }<50 \% \\
\text { del área }\end{array}$ & $\begin{array}{c}25 \%<\text { pérdida de } \\
\text { resistencia }<50 \%\end{array}$ & $\begin{array}{c}25 \%<\text { resistencia } \\
\text { residual }<50 \%\end{array}$ & REGULAR \\
\hline $\mathrm{C} 3$ & $\begin{array}{c}\text { deterioro } \geq 50 \% \\
\text { del área }\end{array}$ & $\begin{array}{c}\text { pérdida de } \\
\text { resistencia } \geq 50 \%\end{array}$ & $\begin{array}{c}\text { resistencia residual } \\
\geq 50 \%\end{array}$ & MALO \\
\hline
\end{tabular}

Cuadro 5. Clasificación según inspección visual e inspección no destructiva.

do se realiza trabajo en campo. Los criterios utilizados para clasificar la inspección visual, gráficas del resistógrafo y la resistencia residual se resume en el Cuadro 5.

Las estructuras de madera evaluadas la conforman vigas, viguetas y columnas, elementos estructurales que varían en sus dimensiones y en su funcionalidad por lo que los resultados se presentan de acuerdo a cada estructura.

Los resultados presentados en los Cuadros 1,2 y 3 resumen la evaluación realizada a cada elemento de madera estructural del estudio, teniendo en cuenta la inspección visual y las técnicas no destructivas como dos parámetros que se complementan para estimar el estado actual de las estructuras. Por otro lado, con la inspección no destructiva se determinó la resistencia de la madera mediante el cálculo del módulo de elasticidad residual y el módulo de rotura residual, dichos parámetros se compararon para dar resultado al diagnóstico. Por último, se comparó estos dos tipos de diagnóstico resultando en la estimación de la clase resistente actual de la madera. En el caso de las columnas, solo se realizó la inspección visual de la estructura, no se pudo realizar el cálculo de los parámetros de resistencia debido a su avanzado grado de deterioro.

\section{Discusión}

La metodología de inspección de estructuras de madera de Arriaga et al. (2002) a pesar de abarcar todas las pautas fundamentales para una exitosa evaluación de maderas de antiguas no se adapta por completo a las características observadas en in situ de estas estructuras, por lo que se modificó, sistematizo y ordeno secuencialmente los pasos a realizar en una evaluación de estructuras de madera como se encuentran en nuestro país.

La propuesta de clasificación en tres categorías (C1, C2 y C3) resulta de la necesidad de no contar con una normativa nacional o extranjera que considere los agentes bióticos como factores fundamentales de la pérdida de resistencia de la madera, actualmente la normativa (UNE 56.544, DIN 4074, NF B 52-001 y NTP 251.104) para clasificación visual de la madera solo se aplica para maderas nuevas y de escuadrías pequeñas.

Las estructuras de madera evaluadas como las vigas, viguetas y columnas se evaluaron de manera distinta cada una por presentar limitantes particulares como sus dimensiones y el comportamiento con respecto al trabajo que realizan en obra. Coincidiendo con Esteban (2003), se tomó en cuenta, en caso de las vigas, evaluar la estructura en tramos de 1 metro debido a que el elemento de madera presenta variaciones en la resistencia y el deterioro del mismo a lo largo de la misma.

Respecto a la clasificación visual de la madera, luego de homologar las normas extranjeras y la norma nacional comparando parámetros similares entre normas (Cuadro 4), solo se logró tener como parámetro restrictivo los de- 

en monumentos hitóricos - Caso Hotel El Comercio

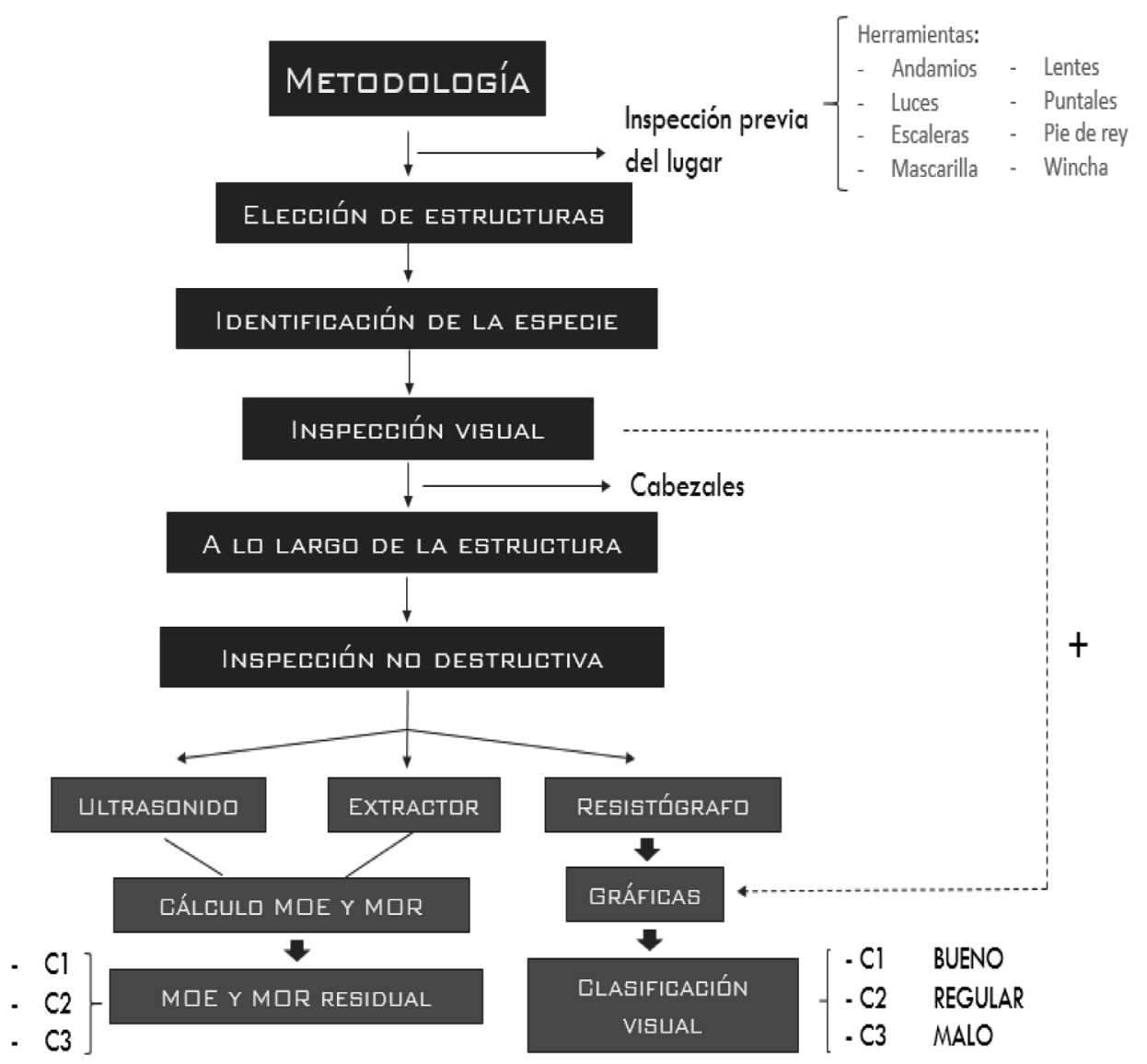

Figura 7. Propuesta metodológica para la evaluación de estructuras de madera antiguas.

fectos mecánicos de la madera debido a que las características anatómicas de las estructuras no se lograron observar a simple vista. Tampoco se consideró los defectos bióticos en los parámetros para la clasificación debido a que no eran aplicables al caso en estudio. Es por ello que se propuso y utilizo la clasificación descrita en el Cuadro 5.

En relación a la validación de los equipos no destructivos mediante los parámetros de MOE, MOR y densidad, el coeficiente de determinación para cada uno de ellos proporciona valores apropiados en comparación con lo mencionado por Benítez (2009), sin embargo, hay que tener en cuenta que en el estudio se han hecho ensayos con un número limitado de repeticiones y es conveniente hallar una relación específica para la madera en estudio.

\section{Conclusiones}

La metodología propuesta para la evaluación de estructuras de madera antigua, empleando las técnicas de inspección visual y equipos no destructivos, contribuirá en la obtención de resultados más satisfactorios para la localización 
de las regiones potencialmente atacadas en estructuras de madera que aún se encuentran en servicio.

La clase resistente actual de las estructuras evaluadas (vigas, viguetas y columnas) se estima que se encuentran $50 \%$ por debajo del Grupo Estructural C, proponiéndose dentro de este grupo 3 categorías de acuerdo a su estado biótico y resistencia residual.

La clasificación propuesta para la estimación del estado actual de la madera puede servir como referencia para el diagnóstico in situ de estructuras de madera en monumentos históricos.

Las normativas evaluadas no se ajustan a la realidad de las estructuras inspeccionadas, en su mayoría castigan la calidad de la madera por sus defectos anatómicos y mecánicos, condición que ya no afecta la resistencia actual.

\section{Agradecimientos}

A la Pontificia Universidad Católica del Perú (PUCP) por el interés que tuvieron en la realización de esta investigación, al Mg. Sc. Daniel Torrealba y la Ing. Erika Vicente. Y a la Facultad de Ciencias Forestales de la Universidad Nacional Agraria La Molina (UNALM).

\section{Bibliografía}

Acuña, L; Basterra, A; Casado, M; López, G; Ramón-Cueto, G; Relea, E; Martínez, C; González, A. 2010. Aplicación del resistógrafo a la obtención de la densidad y la diferenciación de especies de madera. Materiales de construcción 61(303): 451-464.

Álvarez, L; Basterra, A; Casado, M; Acuña, L. 2005. Aplicación del Resistógrafo al diagnóstico de elementos singulares en estructura de madera. I Jornadas de Investigación en Construcción. TOMO I. Grupo de Investigación en Estructuras y Tecnología de la Madera de la Universidad de Valladolid. $11 \mathrm{p}$.

Arriaga, F; Peraza, F; Esteban, M; Bobadilla, I; García, F. 2002. Intervención en estructuras de madera. Madrid. $58 \mathrm{p}$.
Basterra, A; Casado, M; Acuña, L; Pinazo, O. 2006. Techniques of pseudo-Non Destructive Testing on structural Wood, by extraction of screws. ReCoPar 2:37-59.

Benítez, J. 2009. Valoración del deterioro de la madera y la efectividad de su consolidación, midiendo la frecuencia fundamental de oscilación. Estudio experimental aplicado a la tablazón de los artesonados de la Iglesia de San Francisco, Quito, Ecuador. Tesis Doctoral. 110 p.

Chavesta, M; Acevedo, M; Cano, JC. 2012. Estudio anatómico e identificación de especies forestales. Evaluación estructural y especificaciones técnicas de las maderas del Hotel El Comercio, Centro de Lima. Centro de Producción Forestal - Universidad Nacional Agraria La Molina (UNALM). Perú. 2 p.

Divos, L; Nemeth, L; Bejó, L. 1999. Evaluation of the wooden structure of a baroque palace in Papa, Hungary. Proc. of 11th International Symposium on Nondestructive Testing of Wood. $8 \mathrm{p}$.

Esteban, M. 2003. Determinación de la capacidad resistente de la madera estructural de gran escuadría y su aplicación en estructuras existentes de madera de conífera. Tesis Doctoral. Universidad Politécnica de Madrid. 195 p.

(INC) Instituto Nacional de Cultura. 2007. Memoria Descriptiva: Proyecto de Emergencia "Consolidación de muros del $2^{\circ}$ Sector del Monumento Histórico Antiguo Hotel Comercio". Lima. Perú.

Íñiguez, G. 2007. Clasificación mediante técnicas no destructivas y evaluación de las propiedades mecánicas de la madera aserrada de coníferas de gran escuadría para uso estructural. Tesis Doctoral. Universidad Politécnica de Madrid. $176 \mathrm{p}$.

Lombillo, I.; Villegas, L. 2006. Metodología No Destructiva aplicadas a la rehabilitación estructural del patrimonio. Universidad de Cantabria, Santander. 23 p.

Norma del Reglamento Nacional de Edificaciones. 2006. E.010. Lima. Perú.

NTP (Norma Técnica Peruana). 2011. Madera aserrada para uso estructural. Clasificación 

en monumentos hitóricos - Caso Hotel El Comercio

visual y requisitos. NTP 251.104.1998. Lima. Perú.

Norma DIN 4074. 1989. Parte 1. Clasificación de la madera aserrada para uso estructural. Alemania.

Norma UNE 56.544. 2001. Clasificación visual de la madera aserrada para uso estructural: madera de coníferas. España.

Norma NF B 52-001. 1998. Reglas de utilización de la madera en la construcción: Clasificación visual para su empleo en estructuras para las principales especies coníferas y frondosas. Francia.

Sandoz, J.; Benoit, Y. 2006. Acousto-Ultrasonic Non-Destructive Evaluation of Historical Wooden Structures. In Aguilar, R; Torrealva, D; Moreira, S; Pando, M; Ramos, LF (eds.). Structural Analysis of Historical Constructions, New Delhi, p. 519-526.

Sotomayor, JR.; Cruz de León, J. 2004. Resistencia mecánica residual en madera estructural deteriorada. Revista Ciencia Nicolaita 37: 157175.

Presentado: 28/02/2018

Aceptado: 26/09/2018

Publicado en línea: 28/12/2018 